\title{
Kulturelle Vielfalt und Integration im Roman «Zafira, ein Mädchen aus Syrien» von Ursel Scheffler
}

\author{
Ihab Mokhtar Khalaf Abdel-Hafiz \\ Doktorand, Minia Universität, Ägypten \\ rishdan2010@yahoo.com
}

Received: February 21, $2021 \quad$ Accepted: April 24, $2021 \quad$ Published: June 30, 2021

Although intercultural learning can be seen as a natural
answer to the modern multicultural society, the topic of "the
other" / "the foreigner" is becoming more relevant in
connection with the 2015 refugee wave in Germany.
According to UNICEF estimates, Germany took in more
than 300,000 Syrian refugee children that year alone, most
of whom were later divided into regular classes in various
federal states. The coexistence of different cultures in the
classroom is a challenge for teachers and children in the host
community as well as refugee children. Multicultural and
problem-oriented children's literature can be used to address
this new situation in the classroom. Cultural alienation, war,
and escape have been addressed in many contemporary
works by famous children's book authors and told in a
simple way for children. Literary impressions enable you to
expand your own perspective on the world and learn to
interpret it in a multifaceted way. Literature helps young
recipients orient themselves better in culturally
heterogeneous everyday life. By reading books, children can
discover the world from different angles. Literary works
stimulate their imaginations and support recipients in
developing empathy. Books of children's literature on this
topic are sometimes available in stores. They contain many


suggestions for teachers on how to deepen the said motivation with exercises and activities. It is important to choose such readings carefully. Prejudice and racism are the "worst enemies" of integration.

Keywords integration, intercultural learning, efficiency of cultural interaction

\begin{abstract}
Obwohl interkulturelles Lernen als eine selbstverständliche Antwort auf die moderne, transkulturelle Gesellschaft betrachtet werden kann, erlangte das Thema der/die „Andere“ / „Fremde“, im Zusammenhang mit der Flüchtlingswelle von 2015 in Deutschland, zusätzlich an Aktualität. Die Koexistenz der verschiedenen Kulturen in den Klassen ist für die LehrerInnen, Kinder der Aufnahmegesellschaft und die Flüchtlingskinder eine Herausforderung. Zur Thematisierung dieser neuen Situation im Klassenverband bietet sich interkulturelle und problemorientierte Kinderliteratur an. Kulturelle Fremdheit, Krieg und Flucht wurden in diversen zeitgenössischen Werken namhafter Kinderbuchautoren aufgenommen und kindgerecht erzählt. Literarische Eindrücke ermöglichen, die eigene Sichtweise auf die Welt zu erweitern und
\end{abstract} facettenreich deuten zu lernen. Literatur hilft jungen Rezipienten, sich in dem kulturell heterogenen Alltag besser orientieren zu können. Durch das Lesen von Büchern können Kinder die Welt aus unterschiedlichen Blickwinkeln entdecken. Literarische Werke fördern ihre Vorstellungskraft, Fantasie und unterstützten die Rezipienten bei ihrer Entwicklung von Empathie. Dies gilt nicht nur für Bücher, sondern generell für Literatur im weitesten Sinne. Im Handel sind teilweise Begleithefte zu dieser themenspezifischen Kinderliteratur vorhanden. Sie beinhalten für Lehrpersonen viele Vorschläge, wie das angesprochene Motiv mit Übungen und Aktivitäten vertieft werden kann. Es ist wichtig, diese Lektüren mit viel Bedacht auszuwählen. Vorurteile, und Rassismus sind die ,schlimmsten Feinde“ der Integration.

Schlüsselwörter: Integration, interkulturelles Lernen, interkulturelle Kompetenz

\title{
1. Einleitung
}


Im Jahre 2011 begann ein Bürgerkrieg in Syrien, der sich zu einem jahrelangen Krieg entwickelte und bis ins Jahr 2021 andauert. In den Jahren 2015 und 2016 versuchten mehr als eine Million verzweifelter syrischer Kriegsflüchtlinge Europa zu erreichen. Viele Flüchtlinge kamen in Deutschland an (vgl. Strelow 2019, S. 7).

Deutschland war nicht auf einen solchen Flüchtlingsstrom vorbereitet. Die Aufnahme der vielen Menschen aus einem anderen Kulturkreis sorgte in der Gesellschaft für Unruhe, es kam zu Auseinandersetzungen und Konflikten. Die Behörden und die Politik waren mit der Situation überfordert. Spannungen entstanden auch, da die Flüchtlinge falsche Vorstellungen von dem Aufnahmeland hatten und enttäuscht auf die Realität reagierten, die sie nun antrafen. Durch die unterschiedliche Auffassung von Normen und Werten kam es teilweise auch zu inadäquatem Verhalten. Zudem waren viele der Flüchtenden stark traumatisiert durch die erlebten Kriegsgeschehnisse und die Flucht. Verständigungsschwierigkeiten kamen als weiteres erschwerendes Element hinzu (vgl. Zimmermann, 2015, S. 744).

In der Aufnahmegesellschaft entstanden zunehmend Vorurteile gegenüber den syrischen Flüchtlingen un es kam zu rassistischen Reaktionen. Neben den zuvor erwähnten Konflikten waren oft Missgunst und Angst die Treiber für dieses Verhalten. In sämtlichen Medien wurde tagtäglich über die Flüchtlingsproblematik berichtet. Viele deutsche Autoren, sahen sich genötigt zu diesem brisanten omnipräsenten Thema Stellung zu beziehen. Neben verschiedenster Fachliteratur wurden auch fiktive Geschichten für Jung und Alt geschrieben, die das Thema der Flüchtlinge aus verschiedenem Blickwinkel literarisch beleuchten, zum Beispiel der Roman «gehen, ging, gegangen» von Jenny Erpenbeck, „Sommer unter schwarzen Flügeln“ von Peer Martin, „Bestimmt wird alles gut" von Kirsten Boie und «Djadi, Flüchtlingsjunge» von Peter Härtling (vgl. Gündüz \& Asutay, 2020, S. 1).

Jeder Schriftsteller hatte seine eigene Motivation, weshalb er ein Werk zur Flüchtlingsthematik verfasste. Die Buchautorin Ursel Scheffler machte als Kind selbst Kriegserfahrungen. In der aktuellen Flüchtlingskrise Deutschlands besuchte sie Aufnahmezentren, um den 
Kindern Deutsch näher zu bringen. Bei Lesungen in Schulen bemerkte sie, dass den Flüchtlingskindern nicht immer die nötige Zuwendung geschenkt wird. Das Buch «Zafira, ein Mädchen aus Syrien» richtet sich an 10-jährige Rezipienten und nimmt die Alltagssituationen eines normalen Schulalltags auf. In dieser Geschichte zählen 2 syrische Mädchen zu den Protagonistinnen. Diese zwei Mädchen aus Syrien haben in ihrer Heimat sehr unterschiedliche Erziehungsstile genossen. Zafira, die Hauptfigur des Romans, wurde sehr liberal erzogen. Ihr Vater ist Chauffeur und ihre Mutter Englischlehrerin. In Syrien durfte sie schon schwimmen lernen, springen und mit den Jungs auf die Bäume klettern. Alima wurde sehr arabisch traditionell erzogen. Sie hatte weniger Freiheiten. Ihr Vater will vorerst auch in Deutschland nicht auf die alten Traditionen und Sitten der Heimat verzichten. Für Zafira war es einfacher als für Alima, sich den deutschen Werten und Normen anzupassen. Sie darf Fahrrad fahren, macht beim Schwimmunterricht mit und besucht die Geburtstagsparty ihrer deutschen Freundin Anna. Alima muss sich solche Aktivitäten, teilweise gegen den Willen des Vaters, erkämpfen. Das Zusammentreffen verschiedener Kulturen verändert fast immer familiäre Rollen und Hierarchien und verlangt von den geflüchteten Familien, sich mit den kulturellen Unterschieden zu beschäftigen.

\section{Interkulturelle Kompetenz.}

Es gibt keine einheitliche Definition des Begriffs „Interkulturelle Kompetenz", sondern es existieren verschiedene Modelle und Umschreibungen. Alle Erklärungen deuten an, dass interkulturelle Kompetenz ein Schlüsselbegriff ist, der die Begegnung und das Handeln zweier Individuen beschreibt, die aus verschiedenen Kulturen oder sonst aus einem anderen Kontext stammen und nicht durchwegs dasselbe Verständnis von Normalität teilen. Das Erlernen dieser Kompetenz, wird in Anbetracht der Transkulturalität vieler Länder und der Globalisierung, von immer größerer Bedeutung. In der Definition von Nadia Bleil wird ersichtlich, dass die Interkulturelle Kompetenz auf die Soziale Kompetenz aufbaut:

Interkulturelle Kompetenz ist eine besondere Form sozialer Kompetenz, die sich ausschließlich in wahrnehmbaren Verhaltensweisen äußert. Sie ist nötig, um mit Menschen anderer kultureller Prägung auf eine Art in Beziehung zu 
treten, die für alle Beteiligten akzeptabel oder aber zumindest nicht inakzeptabel ist. Sie wird in allen interkulturellen sozialen Situationen mehr oder weniger relevant und erfordert ein besonderes Verhaltensrepertoire. (Bleil, 2006, S. 183)

Interkulturelle Kompetenz setzt die Erkenntnis voraus, dass die eigene kulturelle Prägung bei der Wahrnehmung des Fremden eine wichtige Rolle spielt. Fremde Werte und Normen werden deshalb unterschiedlich empfunden, interpretiert oder aufgenommen. Grundsätzlich ist es spannend und lehrreich, andere Kulturen kennenzulernen. Es festigt die eigene Persönlichkeit und erweitert den eigenen Horizont.

Voraussetzungen dafür sind echtes Interesse, Respekt gegenüber anderen Kulturen und Bemühungen, sich möglichst vorurteilsfrei auf etwas Neues einzulassen. Ebenso bedarf es Toleranz, die Fähigkeit zur Empathie und die Bereitschaft, Differenzen anzuerkennen und zu akzeptieren. Die Einstellung zu dem Fremden kann sich ändern, indem es mit der Zeit vertraut wird und nicht mehr als fremd wahrgenommen wird. Die Interkulturelle Kompetenz umfasst jedoch nicht nur den sprachlichen Austausch, sondern auch nonverbale Formen der Kommunikation. Verhaltensvorschriften und Regeln müssen beachtet werden, denn das spezifische Konzept der Menschen einer anderen Kultur setzt sich zusammen aus der Art ihrer Wahrnehmung, ihres Denkens, Fühlens und Handelns. Wer diese Punkte verstanden und verinnerlicht hat, gilt als interkulturell kompetent (vgl. Mayr, 2012, S. 22).

\section{Interkulturelles Lernen}

Interkulturelles Lernen ist im ausgehenden 20. Jahrhundert so wichtig geworden, dass es laut einem Beschluss der Kultusministerkonferenz 1996 offiziell in die Lehrpläne für die Schüler Deutschlands aufgenommen wurde. Die neu formulierten Leitsätze richten sich nicht nur an die Angehörigen der Majorität, sondern auch an diejenigen der Minorität. Ziel ist es, ein konstruktives Miteinander der Schüler und Schülerinnen zu fördern und das Interesse und die Neugier an der kulturellen Vielfalt zu wecken. Hier ist eine kleine Begebenheit, die die Notwendigkeit des interkulturellen Lernens verdeutlicht:

Ein Hund sieht eine Katze, die mit dem Schwanz wedelt. Freudig erwidert er den freundlichen Gruß, nicht wissend, dass die Katze das Signal als Warnung 
versteht: „Stopp! Nicht weiter, sonst zeig ich dir die Krallen!“ Das Ende der Geschichte kennt jeder: die Katze kratzt, der Hund beißt und die Feindschaft nimmt ihren Lauf. (Breuer\& de Bartha, 2012, S. 32)

Als Vergleichsbasis für das Fremde oder das Andere dient das Eigene, die «Ich»-Identität und die kollektive Identität eines Individuums. Durch spielerische Übungen, Austausch und Lektüren werden die Schülerinnen und Schüler angehalten, ihre Einstellungen und Verhaltensweisen zu ethnischen Grundsätzen der Humanität und den Prinzipien von Freiheit und Verantwortung zu reflektieren. Dieser Prozess trägt zur Herausbildung der eigenen Identität bei und führt dazu, individuelle Strukturen und Ordnungen zu verinnerlichen. Diese begrenzten Ordnungen sind die Voraussetzung, dass Fremdes als fremd wahrgenommen wird, da es sich dem Zugriff des eigenen Ordnungssystems entzieht. Bernhard Waldenfels schreibt dazu:

So viele Ordnungen, so viele Fremdheiten. Das Außerordentliche begleitet die Ordnungen wie ein Schatten. (Waldenfels, 1997, S. 33)

In der Pädagogik realisierte man, dass der, die, das Fremde schon immer eine Herausforderung für den Menschen darstellt, da Kinder und Erwachsene bezüglich des Fremden immer hin- und hergerissen zwischen Gefühlen der Verlockung, Faszination oder Angst und Bedrohung sind. Den Schülern und Schülerinnen muss vermittelt werden, dass eine friedliche Verständigung aller Ethnien Deutschlands jedoch nur funktionier, wenn die demokratischen Werte hochgehalten werden, denn Toleranz und Akzeptanz hören dort auf, wo demokratische Grundregeln und Menschenrechte missachtet oder verletzt werden (vgl. Ahrends \& Nowitzki, 1997, S. 66).

Folgende Grundlagen fördern interkulturelles Lernen (vgl. Nieke, 2008, S. 75):

- Interkulturelles Lernen verlangt eine Auseinandersetzung mit der eigenen kulturellen Identität, dem Erfassen der eigenen Werte, Normen und Deutungsmuster. Das Eigene und das Fremde stehen in einer direkten Beziehung zueinander, wie auch das «Selbstverstehen» und «Fremdverstehen» voneinander untrennbare Begriffe sind. Zudem 
braucht es das Bewusstsein, dass die eigene Sichtweise keine universale Gültigkeit besitzt.

- Nötig ist fächerübergreifendes, vorurteilfreies Vermitteln von Wissen über fremde Kulturen. Neu Erlerntes sollte durch praktische Aktivitäten wie Z.B. Rollenspiele, Diskussionen oder bildnerisches Gestalten vertieft werden. Diese Lernarrangements helfen bei der Auseinandersetzung mit dem Fremden und beim Abbau allfälliger negativer Gefühle gegenüber Personen mit Migrationshintergrund.

- Die Differenzen können so wahrgenommen und erlebt werden. Wenn sie zu Spannungen führen, muss erlernt werden, pragmatisch damit umzugehen.

- Das Einüben von Formen vernünftiger und friedlicher Konfliktbewältigung, anhand von gemeinsam vereinbarten Regeln, ist sehr wichtig. Entstehen Konflikte aufgrund von sich widersprechenden Werteüberzeugungen, sollten nicht die Handlungsvorschriften der Majoritätskultur unweigerlich Recht bekommen. Eine gemeinsame Konfliktregelung erfordert von allen Beteiligten das Bemühen, den eigenen Standpunkt aus der Sicht des oder der anderen zu betrachten.

- Unverständnis, Unwissenheit und Vorurteile können zu Rassismus führen. Deshalb sollte diese Themen ausführlich erläutert und Abwertungsmechanismen aufgezeigt und besprochen werden. Es muss klar sein, dass Abwertungen und Feindseligkeiten gegenüber Menschen, die sich vor allem in körperlichen Merkmalen unterscheiden, gesellschaftlich nicht akzeptiert sind.

- Erlernt werden sollte zudem die Fähigkeit, seine eigene Meinung zu vertreten, ohne dabei die Sichtweise des Anderen ausser Acht zu lassen. Konsensfähigkeit anstelle von Ethnozentrismus soll gefördert werden.

Diverse Projekte von Organisationen wie zum Beispiel das Kooperationsprojekt der Diakonie Düsseldorf «Bilder im Kopf», das interkulturelle Kinderbuchprojekt «Eene Meene Kiste!» oder der Verband binationaler Familien und Partnerschaften weisen auf ihren Websites auf wertvolle, Rassismus freie Kinderliteratur hin. Zudem geben die Kultusministerien der verschiedenen Bundesländer Hinweise 
für geeignete Literatur, die dem Alter der Schulkinder entsprechend im Unterricht eingesetzt werden kann. Die Gefahr beim Lesen von ungeeigneter Literatur besteht darin, dass negative oder schlechte Bilder im Gedächtnis impliziert werden. Es ist schwer, diese Bilder später zu korrigieren.

\section{Inhaltsangabe und Interpretation des Romans «Zafira. Ein Mädchen aus Syrien» von Ursel Scheffler.}

Das Werk mit dem Titel «Zafira. Ein Mädchen aus Syrien» wurde von der deutschen Schriftstellerin Ursel Scheffler geschrieben. Der Roman gehört zur deutschen zeitgenössischen Kinderliteratur. Der Roman wurde im Jahre 2016 im «Hase und Igel» Verlag in München veröffentlicht. Es ist ein schmales Buch von 96 Seiten. Auf dem bunten Buchumschlag sieht man fröhliche Schulkinder mit ihren Schulranzen. Der Name der Protagonistin - Zafira - und das Abbild des Mädchens nehmen einen grossen Teil des Buchdeckels ein. Der Hintergrund ist in leuchtendem Gelb gehalten. Das Buch enthält farbige Bilder, die von Frau Jutta Timm gestaltet worden sind. Die Bilder der tragischen Binnengeschichte sind mit dunkler Farbe hinterlegt.

Der Roman schildert die Geschichte eines Mädchens namens Zafira, das mit seiner Mutter, Nesrin, nach dem Krieg in Syrien nach Deutschland flüchtet. Sie wollen Hamburg erreichen, denn dort lebt die Schwester des Vaters Zafiras mit ihrem Mann.

Der Roman beginnt in einem Schulzimmer Hamburgs. Die Schüler haben ein Volleyballspiel gegen die Parallelklasse gewonnen und sind voller Freude. Sie sind die Helden, doch jetzt steht ihnen noch eine andere Herausforderung bevor, die sie gewinnen sollten. Das nächste Ziel ist die Inklusion ihrer neuen Mitschülerin, die Zafira heißt. Frau Scheffler übernimmt hier indirekt die Rolle von Frau Bartos, der Klassenlehrerin. Sie stellt Zafira den anderen Schülern vor, ohne Mitleid für sie zu wecken. Sie ist bedacht darauf, dass die Kinder Zafira mit Interesse, Respekt und auf Augenhöhe entgegentreten. Frau Bartos erklärt, dass Zafira aus einem Land mit einer uralten Kultur kommt und eine Sprache spricht, die von über 200 Millionen Menschen gesprochen wird. 
Die ersten zwei Kapitel spielen sich im Schulbereich ab und die Rede ist primär von Zafira, ihrer Sprache und Sprachentwicklung, ihrer Kultur und ihrer Heimat.

Das 3. Kapitel beschreibt Zafiras Annas, Klassenkameradin, Geburtstagsparty, zu der auch Zafira eingeladen ist. Hier stellen die anderen Mädchen Zafira Fragen über ihr früheres Leben in Syrien. Diese basieren auf Stereotypen, die die Mädchen von Syrien haben. Sie fragen zum Beispiel, ob Zafira in Syrien im Zelt gelebt hat, das Wetter in der arabischen Wüste sehr heiß ist oder ob sie ein Kamel besessen habe. Zafira beantwortet und korrigiert lachend diese Fragen. Sie sagt, dass sie nur einmal in einem Zelt lebte, das war aber in Deutschland und nicht in Syrien. Als Annas Mutter sie auf der Party fragt, was sie trinken wolle, antwortet sie: „Das was alle trinken“. Zafira will damit kundtun, dass sie anpassungsfähig ist.

Im 4. Kapitel erfährt der Rezipient wie stark Zafira traumatisiert ist. Die Knallfrösche, die die Jungs knallen lassen versetzen sie in Panik, so dass Anna Zafira kaum beruhigen kann. Später erfahren die Kinder, dass Zafira wegen den Kriegserfahrungen in ihrem Heimatland so empfindlich auf das Knallen und Lärm reagiert. Der Junge, der sich daraufhin bei Zafira entschuldigt, wird im Buch als mutig bezeichnet. Den Rezipienten wird dadurch vermittelt, dass es positiv und lobenswert ist, sich zu entschuldigen, wenn man einen Fehler eingesehen hat.

Ab Kapitel 5 beginnt die Binnengeschichte. Sie wird nicht von der Lehrerin oder der Mutter von Zafira erzählt, sondern von dem auktorialen Erzähler. Sie steht im Präteritum geschrieben. Es ist eine Rückblende auf Zafiras Leben in Syrien nach Kriegsbeginn und auf die Flucht nach Deutschland. Sie endet mit der Katastrophenmeldung einer Zeitung. Nicht alle Passagiere des vor der italienischen Küste gesunkenen Flüchtlingsschiffs Hope konnten gerettet werden. Der Ausgang dieses Unglücks für Nesrin und Zafira wird hier nicht thematisiert, sondern zu einem späteren Zeitpunkt erwähnt. In diesem Abschnitt erfahren die jungen RezipientInnen die schrecklichen Auswüchse des Krieges, der Zerstörung und vom vielen Leid für die Zivilbevölkerung, das der Krieg mit sich bringt. 
Ab Kapitel dreizehn beginnt der zweite Teil der Rahmengeschichte. Zafira ist jetzt nicht mehr die „Neue“, sie spricht inzwischen, dank ihrer positiven Einstellung und der Unterstützung vieler Mitmenschen, gut Deutsch und integriert sich immer besser in die neue Heimat. Neun Monate sind seit ihrem ersten Schultag vergangen. Frau Bartos hat zwei weitere Flüchtlingskinder für ihre Klasse zugeteilt bekommen. Auch sie sind aus Syrien, aus Homs und heißen Alima und Samir. Zafira unterstützt die zwei Neuankömmlinge und lädt sie mit ihrer Familie zu sich nach Hause ein. Dieses Treffen der beiden syrischen Flüchtlingsfamilien beinhaltet nochmals eine Art Binnengeschichte. Diese nimmt Bezug auf das Fluchterlebnis Abduls Familie. Ihre Flucht führte sie über die sogenannte Balkanroute. Abdul verkörpert in diesem Roman den typischen arabischen Mann. Er ist das Oberhaupt der Familie und achtet darauf, dass die traditionellen Regeln eingehalten werden. So bestimmt er auch über die Freiheiten seiner Frau und Tochter.

\section{Die literarische Inszenierung einer deutschen Kultur im Gegensatz zur syrischen Herkunft unter dem Aspekt kultureller Vielfalt}

Die Hauptfiguren des Romans Zafira, Alima und Anna bieten sich jungen Leserinnen und Lesern als Identifikationsfiguren an. Die Lehrerin spielt bei den Erwachsenenfiguren eine tragende Rolle. Sie wirkt sehr einnehmend, motivierend und lobt ihre Schüler. Anstatt die Schüler zurechtzuweisen oder zu bestrafen setzt sie erfolgreich auf Aufklärung und positive Verstärker. Anhand der vier Hauptfiguren des Romans wird im Folgenden mehr auf das Zusammenleben zwischen den verschiedenen Kulturen eingegangen:

Zafira ist die Protagonistin dieses Romans. Sie kommt aus der Stadt Aleppo in Syrien. Sie ist ca. 10 Jahre alt, schlank, hat kastanienbraunes gelocktes Haar und dunkelbraune Augen (Scheffler, 2016, Z., S. 10). Sie ist größer als ihre deutsche Klassenkameradin Anna.

Mit ihrer Mutter Nesrin ist sie nur mit den nötigsten Habseligkeiten und Stofftier Pascha über die Türkei, das Mittelmeer und Italien nach Hamburg geflüchtet (Z. 50/51). Mittellos kommen sie hier an. Ihr ganzes Geld bezahlten sie den Schleppern und Fluchthelfern. (Z. 59) Der schreckliche Krieg in Syrien und die schwierige Flucht nach Deutschland 
prägen Zafira. Nur dank ihren Schwimmkenntnissen überlebte sie die Flucht (Z. 78), denn der Kapitän musste den schrottreifen Frachter „Hope“ vor der Küste Italiens auf Grund laufen lassen (Z, 72). Das stark überfüllte Rettungsboot, das sie an Land bringen sollte, kenterte kurz vor der italienischen Küste und sank. Mit viel Glück erreichten danach Zafira und ihre Mutter mit dem Zug Deutschland. Tante Selina und ihr Mann holten sie in München mit dem Auto ab (Z. 82).

In Hamburg lebt Zafira zuerst in einem Container und dann mit anderen Flüchtlingen in einem Aufnahmezentrum. Als Zafira in Deutschland eingeschult wird, wirkt sie ängstlich und verunsichert (Z. 10f). Dank der netten Lehrerin, die sie stärkt und auf ihre spezielle Situation eingeht und der sozialkompetenten Mitschülerin Anna, lernt Zafira schnell ihre ersten Sätze auf Deutsch zu formulieren und wird immer mutiger (Z. 13). Sie geht gerne in die Schule und liebt vor allem die Schulfächer Mathe, Sport, Kunst und Musik (Z. 20). In diesen Fächern spielt die Sprache eine untergeordnete Rolle. Dank Anna findet sie schnell Anschluss an die neuen Schulkollegen-innen. Sie freut sich sehr über Annas Einladung zur Geburtstagsparty. Bei diesem Anlass erzählt sie den anderen Mädchen von ihrem früheren Leben in Aleppo (Z. 22) und korrigiert deren falschen Vorstellungen über ihren Alltag in Syrien.

Oft wirkt Zafira aber auch traurig und erschreckt sich an lauten Geräuschen. Das Knallen von Brottüten und der Lärm von startenden Flugzeugen versetzen sie in Angst und Schrecken (Z. 26). Sie versucht zu flüchten, davonzulaufen. Solche Geräusche wecken die schrecklichen Erinnerungen an den Krieg und die Flucht. Ganz klar steigen dann die Bilder der schlimmen Vergangenheit wieder in Zafira hoch und rauben ihr nachts den Schlaf (Z. 28).

Als Zafira schon ein Dreivierteljahr in Hamburg zur Schule geht, spricht sie recht gut Deutsch, so dass sie dem neuen syrischen Flüchtlingsgeschwisterpaar bei deren Integrationsprozess behilflich sein kann (Z. 71). Die zwei Neuen heißen Alima und Samir. Zafira lädt (Z. 74) sie mit ihren Eltern und Kleinkind Mimi (Z. 75) zu sich nach Hause ein. Die Erwachsenen erzählen einander von den schlimmen Erlebnissen auf der Flucht. Die Kinder hören ihnen gespannt dabei zu (Z. 74-79). 
Zafira unterhält sich mit Samir und Alima über das neue Leben in Deutschland und sie stellen fest, dass hier Mädchen dasselbe tun dürfen wie die Jungen. Das finden vor allem die Mädchen sehr gut. Zafira lernte auf der Flucht auch einiges über die Gesetze Europas kennen. Sie weiß zum Beispiel, dass Flüchtlinge nur in dem Land einen Asylantrag stellen dürfen, in dem sie das erste Mal europäischen Boden betreten. Sie hörte auch, dass wegen des massiven Flüchtlingsstromes diese Regel oft gebrochen wurde und viele europäische Länder die Flüchtlinge gerne ohne Kontrolle in andere Länder passieren ließen (Z. 81)

Zafira bekommt von ihrer Tante Selina ein altes Rad geschenkt, da sie schon Fahrrad fahren kann. (Z. 82). Damit besucht Zafira ihre Tante und ihre Freundin Anna oder die Mädchen fahren damit in die Schule. Zafira teilt ihre Freundschaft zu Anna auch mit Alima (Z. 83). Die beiden Freundinnen unterstützen Alima bei ihrem Wunsch Fahrrad fahren zu lernen. Dies gegen den Willen von Alimas Vater. (Z. 85)

Als Zafira eines Nachts wieder nicht schlafen kann und mit ihrem Stofftier Pascha in die Küche geht, trifft sie dort ihre weinende Mutter an. Sie zeigt Zafira ein Zeitungsbild von der zerbombten Stadt Aleppo und spricht mit ihr über die schwierige Situation in der Heimat. Die Mutter macht sich große Sorgen um ihren Mann, ihre Verwandten und Freunde (Z. 89) Nasrin will Zafira nicht weiter mit ihren Ängsten und mit schlechten Nachrichten aus Syrien belasten. Sie freut sich, dass Zafira wieder ein fröhliches Kind ist, das gerne zur Schule geht und fleißig lernt (Z. 91). Sie hegt den Berufswunsch einmal Lehrerin zu werden (Z. 93)

Als an einem Tag die gute Nachricht kommt, dass Zafiras Großeltern den Angriff auf ihr Dorf in den Bergen überlebt hatten und sich zu türkischen Verwandten durchschlagen konnten, freuten sich Zafira sehr darüber (Z. 92) In den nächsten zwei Jahren lernt Zafira mit ihrer Tante Selina die Umgebung von Hamburg kennen. Die Stadt ist für Zafira zur zweiten Heimat geworden. Trotzdem fehlt ihr etwas. Sie vermisst ihren Vater sehr, der ihr von einem Bild über ihrem Bett immer wieder zulächelt. Eines Tages als Zafira sich gerade aufs Fahrrad schwingen und zu Anna radeln will, hält neben ihr ein Taxi. Ein Mann mit weißem Haar und schmalem, blassen Gesicht fragt sie nach dem Haus Nummer 46. Zafira 
wundert sich, dass der unbekannte Mann zu ihrem Wohnhaus gehen will, zeigt es ihm aber bereitwillig (Z. 94)

Als Zafira später wieder nach Hause kommt, empfängt sie ihre Mutter freudestrahlend. Da erst merkt Zafira, dass der Mann mit den weißen Haaren ihr Vater Sinan ist (Z. 95).

Frau Bartos ist eine sehr engagierte und motivierte Lehrerin. Die Schüler mögen sie und ihre spannenden Erklärungsgeschichten. Sie unterrichtet eine 4. Klasse und bereitet ihre Schüler gewissenhaft auf eine neue Mitschülerin vor. Indem sie das Wort „Zafira“ kommentarlos an die Wandtafel schreibt, gewinnt sie schnell die Aufmerksamkeit der Kinder, die darüber rätseln, was dieses Wort wohl bedeutet.

Frau Bartos erklärt, dass dies der arabische Mädchenname der neuen Mitschülerin ist und so viel wie „hell und glänzend“ bedeutet. Sie teilt der Klasse auch mit, dass Zafira ein Flüchtlingskind ist und ihre Heimat Syrien unfreiwillig verlassen musste. Sie fragt die Schüler, was sie über Syrien wissen und erklärt, dass Zafira kein Deutsch spricht. Sich zu verständigen funktioniert aber auch mit Gesten, Mimik und der Klangfarbe der Stimme (Z. 7-10).

Als Zafira mit ihrer Mutter in die Schule kommt, begrüßt sie die beiden sehr freundlich, stellt sie der Klasse vor und platziert Zafira bewusst neben Anna, die schon eine hohe Sozialkompetenz mitbringt. (Z. 11). Dank der guten Atmosphäre im Klassenzimmer und der Unterstützung der Mitschüler-innen, lernt Zafira schnell einzelne Sätze auf Deutsch (Z. 13).

Frau Bartos nimmt das Thema Syrien auf positive Art und Weise in ihren Schulstunden immer wieder auf. So lernen auch Zafiras Klassenkameraden-innen etwas Neues über dieses ferne Land. In einer Mathestunde erklärt Frau Bartos den Schülern, dass die Zahlen, mit denen sie Rechnen, durch die Araber nach Europa kamen (Z. 15-18). Als die Mutter wegen eines Lausbubenstreichs, bei dem sich Zafira sehr erschreckte, in die Schule kommt, wird sie von Frau Bartos ernst genommen. Sie bespricht das Problem am nächsten Tag während einer Mathestunde mit der Klasse. Ohne Sanktionen anzudrohen, merken die 
Jungs, dass sie einen Fehler gemacht haben und entschuldigen sich bei Zafira (Z. 27/28).

Ein Dreivierteljahr später stoßen nochmals zwei syrische Flüchtlingskinder zu der Klasse. Frau Bartos ist stolz, dass alle Schüler etwas toleranter geworden sind und eine kulturelle Sozialkompetenz entwickelt haben. Voller Freude registriert sie, wieviel Zafira in den letzten neun Monaten dazugelernt hat. Sie fragt sich, ob sie selber so schnell Arabisch gelernt hätte (Z. 73).

Anna ist in der 4. Klasse bei Frau Bartos. Der Platz am Pult neben Anna ist frei, da ihre Freundin Iris weggezogen ist. Die Lehrerin beschließt Zafira diesen Platz zuzuteilen. Anna spürt sofort, dass Zafira unsicher ist, steht spontan auf, nimmt Zafira an der Hand und fordert sie freundlich auf, neben ihr Platz zu nehmen (Z. 11/12).

Diese Szene verdeutlicht, dass Anna eine Sozialkompetenz entwickelt hat. Anna und Zafira freunden sich schnell an. Anna teilt mit Zafira ihr Pausenbrot (Z. 16) und lädt sie zu ihrer Geburtstagsparty ein (Z. 21).

Als nach der schönen Geburtstagsfeier die Jungen Zafira mit Knallfröschen erschrecken, wird Anna furchtbar wütend, schimpft mit ihnen und versucht ihre Freundin zu beruhigen (Z. 26). Während Zafira fleißig Deutsch lernt, lernt Anna ein paar Brocken Arabisch (Z. 74). Ein kultureller Austausch findet zwischen den zwei Mädchen statt. Als geraume Zeit später nochmals zwei syrische Flüchtlingskinder zur Klasse stoßen, ist Anna sofort bereit, das Mädchen Alima in ihren Freundeskreis mit aufzunehmen. Sie bittet aber Zafira und Alima, in ihrer Gegenwart Deutsch und nicht Arabisch zu sprechen (Z. 83).

Anna und Zafira helfen Alima beim Erlernen des Fahrradfahrens (Z. 84/85). Anna bekommt sogar von ihrer Cousine ein altes Rad geschenkt, dass sie Alima weitergibt. Annas Vater lässt noch die Bremsen beim Fahrradhändler überprüfen und Annas Mutter kauft eine lustige Klingel fürs Rad. Annas Eltern zeigen sehr viel Offenheit und Hilfsbereitschaft gegenüber den beiden Mädchen aus Syrien. Deshalb verwundert es jetzt nicht, da ihre Tochter Anna in jungen Jahren schon 
ein hohes Maß an Sozialkompetenz entwickelt hat, was sich in ihrer Art des Interagierens mit Zafira ausdrückt.

Alima, ihr Bruder Samir und ihre Eltern Selma undAbdul wohnten in Homs in Syrien. Auch sie mussten wegen des Krieges fliehen und gelangten etwas später als Nesrin und Zafira nach Hamburg. Alima und Samir werden in dieselbe Klasse eingeteilt wie Zafira (Z. 73). Alima ist froh, dass Zafira ihre Muttersprache spricht und in der neuen Heimat schon gut Fuß gefasst hat. Die zwei Mädchen verstehen sich prima. Zafira lädt Alima und ihre Familie zu sich nach Hause ein (Z. 74). Beide Familien freuen sich über das Treffen und tauschen rege ihre Erfahrungen aus. Alima und ihre Familie sind von der Türkei über das Meer nach Griechenland geflüchtet und dann wochenlang zu Fuß bis nach Skopje in Mazedonien gewandert. Dort kam Mimi in einer Klinik zur Welt. Später ging die Flucht mit einem Pferdefuhrwerk und wieder zu Fuß weiter. An der ungarischen Grenze nahm sie jemand, gegen Bezahlung, in einem Möbelwagen mit bis nach Wien. Von dort aus fuhren sie in einem Touristenbus weiter nach Deutschland (Z. 75/76). Samir erinnert sich, dass sie bei der Ankunft in Deutschland fürchterlich froren. Im Aufnahmezentrum, bekam die Familie warme Kleider (Z. 77). Dort halten sie sich nun schon seit drei Monaten auf. Das Leben im Aufnahmezentrum ist nicht einfach. Man hat keine Privatsphäre (Z. 78). Etwas später nach dem positiven Asylbescheid finden sie eine kleine eigene Wohnung (Z. 84). Alima gefällt, dass in Deutschland die Jungen und Mädchen die gleichen Rechte haben und die gleichen Sachen machen dürfen. Dies vor ihrem Vater Abdul zu äußern, verlangt ihr allerdings eine Portion Mut ab. Der Vater will sie daraufhin auch gleich zurechtweisen, aber seine Frau Selma greift besänftigend ein. Selma nimmt Bezug auf ihre aktuelle Situation und meint, dass sie sich an die neuen Werte und Normen der deutschen Gesellschaft anpassen sollten, damit sich die Kinder längerfristig in Deutschland wohl fühlen können. Zudem müssten sie die Sprache der neuen Heimat lernen, damit sie sich verständigen können. Abdul kommentiert diese Aussage nicht.

Alima ist überglücklich, als sie kurze Zeit später, an ihrem Geburtstag, ein paar Sportschuhe und einen Schwimmanzug geschenkt bekommt 
(Z. 79). So wird sie nicht zur Außenseiterin und kann an den sportlichen Aktivitäten, wie alle anderen Kinder, teilnehmen. Alima und Zafira werden dicke Freundinnen, die sich alles anvertrauen. Alima will von Zafira ganz genau wissen, was auf der Flucht vor der italienischen Küste passiert ist. Zafira erzählt ihr, wie schlimm und schwierig diese Situation war. Alima kann verstehen, wie herausfordernd diese Momente waren, zumal sie die gesprochene Sprache nicht beherrschten (Z. 81). Es gibt viele Passagen in der Fluchtgeschichte Zafiras, die Alima sehr vertraut vorkommen, da sie sie selber auch erlebte (Z. 82). Während die zwei Mädchen ein uraltes Brettspiel spielen, gesteht Alima ihrer Freundin, dass sie auch gerne eine deutsche Freundin hätte (Z. 83). Anna ist bereit auch Alima in ihren Freundeskreis aufzunehmen. Ein weiterer Wunsch von dem syrischen Mädchen ist, dass sie gerne Fahrradfahren lernen würde. Ihre Freundinnen helfen ihr, diesen Wunsch umzusetzen und bringen ihr Fahrradfahren bei. Alima stellt sich sehr geschickt an und lernt schnell (Z. 84). Als sie von Annas Cousine sogar noch ein Rad geschenkt bekommt, kann sie an der Fahrradprüfung in der Schule teilnehmen und bekommt am Ende einen Fahrradführerschein ausgehändigt. Den will Alima stolz ihrem Papa zeigen (Z. 87).

Der letzte Satz des Buches wirft das Thema «Heimat» auf. Deutschland wird in dem Roman als «neue Heimat», «fast neue Heimat» oder «neue Heimat vielleicht?» bezeichnet. Diese vorsichtige Formulierung weist darauf hin, dass die Asylanten lange Zeit zwiespältige Gefühle gegenüber ihrem Aufnahmeland hegen. Sie wissen oft noch nicht, ob sie Deutschland als Heimat bezeichnen können oder dürfen.

\section{Fazit}

Die Autorin will den jungen Rezipientinnen mit dieser Geschichte zeigen, dass die Inklusion eines fremden Schülers und einer fremden Schülerin in den Klassenverband nur mit ihrer Intention und Kooperation gelingen wird. Die Geschichte zeigt, dass Zafira und Alima, obwohl sie aus demselben Land kommen, durch verschiedene traditionelle Werte geprägt wurden. Zafira kann rennen, springen, klettern, schwimmen und Fahrrad fahren. Sie durfte dieselben Dinge tun wie die Jungs. Alima blieb dies verwehrt. Deshalb registriert sie diesen kulturellen Unterschied in 
Deutschland sehr schnell und freut sich darüber. Sie erlaubt sich sogar, dies ihrem Vater gegenüber zu äußern und ist bereit für diese neuen Freiheiten zu kämpfen. Sie will auch schwimmen und Fahrradfahren lernen. Über den Erziehungsstil von Zafiras muslimischem Vater erfährt man nichts. Nur anhand der Art und Weise, wie sich Nesrin und Zafira verhalten, kann man ein paar Rückschlüsse ziehen. Zafira genoss mehr Freiheiten in Syrien. Die ganze Familie gestaltet ihr Leben liberaler. In Deutschland werden Zafira und Alima nicht nur gute Freundinnen, sondern sie erstarken auch zu unternehmungslustigen, lebensfrohen Persönlichkeiten.

Die Autorin zeigt mit diesem Buch, welche Voraussetzungen geschaffen werden müssen, damit der Integrationsprozess einen positiven Verlauf nimmt. Die Bemühungen aller sind gefragt. Die Botschaft an die Menschen des Aufnahmelandes lautet, dass sie den Flüchtlingen echtes Interesse entgegenbringen und ihnen auf Augenhöhe begegnen sollten. Für die Geflüchteten ist das Erlernen der Sprache des Aufnahmelandes essentiell, auch wenn man sich ein Stück weit nonverbal unterhalten kann.

Am Ende des Romans heißt es, dass Zafira mit ihren Eltern und der Tante Pläne für die Zukunft in dem neuen Land schmiedet. Und darauf folgt die oft lange quälende Frage: Ist das Aufnahmeland eine neue Heimat vielleicht?

\section{Literaturverzeichnis:}

\section{Primärliteratur:}

Scheffler, U. (2016). Zafira - Ein Mädchen aus Syrien. Hase und Igel Verlag GmbH. (Im Text steht „Z“ bei den Seitenzahlangaben für den Roman: Z. S.)

\section{Sekundärliteratur:}

Ahrends, A. \& Nowitzki, W. (1997). Interkulturelles Lernen in den Lehrplänen. Anregungen für Schule und Unterricht. Ministerium für Bildung, Wissenschaft, Forschung und Kultur des Landes.

Bleil, N. (2006). Interkulturelle Kompetenz in der Erwachsenenbildung: Ein didaktisches Modell für die Trainingspraxis. Lang.

Breuer, J. P., \& de Bartha, P. (2012). Deutsch-französische Geschäftsbeziehungen erfolgreich managen: Spielregeln für die Zusammenarbeit auf Fach- und Führungsebene. 4. Auflage. Springer Verlag. 
Mayr, A. (2012). Interkulturelle Kompetenzen - eine Notwendigkeit in der Elternarbeit? Eine qualitative Studie an Grundschullehrern und-Lehrerinnen aus Wien und Oberösterreich. (Diss. Uniwien).

Nieke, W. (2008). Konzepte Interkultureller Erziehung und Bildung. Interkulturelle Erziehung und Bildung- Wertorientierungen im Alltag, 13-35. Verlag für Sozialwissenschaften.

Strelow, E. (2019). Flüchtlinge in der Altenpflegeausbildung: eine empirische Studie. Springer Verlag.

Waldenfels, B. (1997). Topographie des Fremden - Studien zur Phänomenologie des Fremden 1. Suhrkamp.

\section{Internetquellen}

Gündüz, C. T. \& Asutay, H. (2020). Flüchtlinge in der Kinder- und Jugendliteratur: Am Beispiel von Kirsten Boies ,Bestimmt wird alles gut. Abgerufen am 5. Januar 2021 von: https://bit.ly/3qdZFeX

Hinte, H., Rinne, U., \& Zimmermann, K. F. (2015). Flüchtlinge in Deutschland: Herausforderung und Chancen. Wirtschaftsdienst, 95(11), 744-751. https://doi.org/10.1007/s10273-015-1897-5 